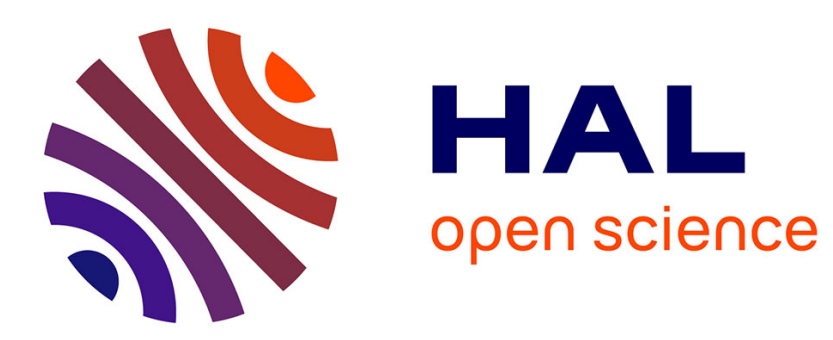

\title{
Robust synchronization of genetic oscillators subjected to cell division and common entrainment
}

\author{
Hafiz Ahmed, Rosane Ushirobira, Denis Efimov
}

\section{To cite this version:}

Hafiz Ahmed, Rosane Ushirobira, Denis Efimov. Robust synchronization of genetic oscillators subjected to cell division and common entrainment. ECC 2016 - 15th annual European Control Conference, Jun 2016, Aalborg, Denmark. hal-01380247

\section{HAL Id: hal-01380247 \\ https://hal.inria.fr/hal-01380247}

Submitted on 12 Oct 2016

HAL is a multi-disciplinary open access archive for the deposit and dissemination of scientific research documents, whether they are published or not. The documents may come from teaching and research institutions in France or abroad, or from public or private research centers.
L'archive ouverte pluridisciplinaire HAL, est destinée au dépôt et à la diffusion de documents scientifiques de niveau recherche, publiés ou non, émanant des établissements d'enseignement et de recherche français ou étrangers, des laboratoires publics ou privés. 


\title{
Robust synchronization of genetic oscillators subjected to cell division and common entrainment
}

\author{
Hafiz Ahmed, Rosane Ushirobira, Denis Efimov
}

\begin{abstract}
Cell division introduces discontinuities in the dynamics of genetic oscillators (circadian clocks, synthetic oscillators, etc.) causing phase drift. This paper considers the problem of phase synchronization for a population of genetic oscillators that undergoes cell division and with a common entraining input in the population. Inspired by stochastic simulation, this paper proposes analytical conditions that guarantee phase synchronization. This analytical conditions are derived based on Phase Response Curve (PRC) model of an oscillator (the first order reduced model obtained for the linearized system and inputs with sufficiently small amplitude). Cell division introduces state resetting in the model (or phase resetting in the case of phase model), placing it in the class of hybrid systems. It is shown through numerical experiments for a motivating example that without common entraining input in all oscillators, the cell division acts as a disturbance causing phase drift, while the presence of entrainment guarantees boundedness of synchronization phase errors in the population. Theoretical developments proposed in the paper are demonstrated through numerical simulations for two different genetic oscillator models (Goodwin oscillator and Van der Pol oscillator).
\end{abstract}

Index Terms-Cell division; Phase resetting; Phase synchronization.

\section{INTRODUCTION}

Several rhythmic/oscillatory processes occur at the cellular level due to the interactions among the different regulators of the genetic regulatory networks. For example, circadian rhythms in mammals result from a transcription-translation-based negative auto regulatory feedback loops [1]. The understanding of the design principles of these oscillations through mathematical modeling, can guide the conception and implementation of new, artificial, genetic oscillators [2]. The interest in analysis and synthesis of genetic oscillators gained the attention of the research community during the last decades due to the importance of genetic oscillators in molecular system biology.

Both natural and artificial genetic oscillators generate sustained periodic oscillation through various feedback loops and external entraining stimulating inputs [3], [4]. These periodic oscillations can be characterized by their frequency, phase and amplitude which are mainly governed by the external entraining inputs, while the phase value is dependent on properties of the oscillator and the characteristics of the entrainment. Since the phase is the only quantity which depends on both the oscillator structure and the nature of entrainement, it has attracted the attention of many

The authors are with Non-A project @ Inria, Parc Scientifique de la Haute Borne, 40 avenue Halley, 59650 Villeneuve d'Ascq, France and CRIStAL (UMR-CNRS 9189), Ecole Centrale de Lille, Avenue Paul Langevin, 59651 Villeneuve d'Ascq, France.

Denis Efimov is with Department of Control Systems and Informatics, University ITMO, 49 avenue Kronverkskiy, 197101 Saint Petersburg, Russia.

The project is supported in parts by ANR project WaQMoS (ANR 15 CE 04 0002), the Government of Russian Federation (Grant 074-U01) and the Ministry of Education and Science of Russian Federation (Project 14.Z50.31.0031).

Hafiz Ahmed is partly supported by the regional council of Nord-Pas de Calais researchers because of its potential application in synthetic biology. A very interesting phenomenon that involves phase is called phase synchronization which happens in networks of oscillators. Phase synchronization is widespread in the nature, for instance in the colony of the smallest free-living eukaryotes, the mammalian circadian pacemaker neural network [5], [6] or networks of neural oscillators [7], [8], [9]. Controlled phase resetting has been studied in [10], [11], [12] and for a population of oscillators in [13].

Phase models are widely used in physics, chemistry, and biology. There exist many methods for the analysis of phase dynamics and phase resetting for a single oscillator. A simple but effective tool in this area is based on PRC [14], [15], [3]. PRC measures phase changes of an oscillator induced by a perturbation applied at different times of the cycle. In this method, the infinitesimal PRC map is calculated for the system linearized around the limit cycle and the linearization is valid for inputs with small amplitudes. If the entraining input is a series of pulses, then a Poincare phase map based on PRC can be calculated to predict the phase behavior [3]. Such a reduced phase model has been used in [11], [13] for pulse amplitude and timing calculation for a controlled phase resetting. Similarly, the reduced phase model has also been used for robust entrainment of circadian oscillators and for synchronization of neural networks [9] etc.

Another interesting problem that has been reported recently in [16] deals with the influence of cell division on the behavior of genetic oscillators. It has been observed that oscillations persist across cell divisions in Repressilator [2] or in dual-feedback synthetic genetic oscillator [17], similarly for circadian oscillations in cyanobacteria cells [18]. In [19], persistence of circadian oscillations in culture fibroblasts under cell division has been demonstrated, and it has been noted that cell division can shift the phase in circadian cycle. A rapid phase decorrelation between daughter cells has been remarked in [20] for oscillations in the p53/Mdm2 system. Since cell division introduces a discontinuity in the oscillator dynamics (that is usually described by a system of nonlinear differential equations), then the analysis of division influence leads to the study of a hybrid or impulsive nonlinear oscillating system, which is a rather complicated problem [21], [22]. In [16] this problem has been investigated using the stochastic simulation approach, and in [23] the geometric phase approach has been adopted from quantum mechanics to explain the phase delay that appears after cell division.

The goal of the present work is to analyze the phase behavior and synchronization under cell division in genetic oscillators using PRC formalism ${ }^{1}$. A motivating example, Goodwin genetic oscillator, is given in section II. Some preliminaries about phase dynamics are summarized in section III. The analysis of cell division influence on the phase dynamics is presented in section IV. An illustration

\footnotetext{
${ }^{1}$ This article represents a conference version of [24]. Here different example has been studied and the text has been rewritten with new references with respect to [24]
} 
by simulations of the obtained results is given in section $\mathrm{V}$.

\section{Motivating EXAMPLE}

Goodwin model of a genetic oscillator, showing that regulatory interactions among genes allow periodic fluctuations to occur, has the following form [25], [16], [26]:

$$
\begin{aligned}
\dot{X}(t) & =u(t)+c_{1} \frac{K^{n}}{K^{n}+Z^{n}(t)}-c_{2} X(t), \\
\dot{Y}(t) & =c_{3} X(t)-c_{4} Y(t), \\
\dot{Z}(t) & =c_{5} Y(t)-c_{6} Z(t),
\end{aligned}
$$

where $X(t), Y(t)$ and $Z(t)$ are the concentrations of clock gene mRNA, clock protein and end product generating the negative feedback loop, respectively; $K>0, c_{i}>0, i=0,1, \ldots, 6$ and $n>0$ are parameters and $u(t) \geq 0$ is the external entraining input. If the parameters are selected properly, then this system for $u(t)=0$ has a locally attracting limit cycle that represents rhythmic behavior of a genetic oscillator with the period $T>0$. It is a continuous-time dynamical system that for any initial conditions $X(0)>0, Y(0)>0$ and $Z(0)>0$ has a continuous positive solution for all $t \geq 0$. To model cell division in (1), it is necessary to introduce an increasing series of time instants $t_{k}>0, k=1,2, \ldots$ with a division at each $t_{k}$. During division, the state variables are resetted [16], i.e. $X\left(t_{k}^{+}\right)=\lambda_{k}^{X} X\left(t_{k}\right), Y\left(t_{k}^{+}\right)=\lambda_{k}^{Y} Y\left(t_{k}\right)$ and $Z\left(t_{k}^{+}\right)=\lambda_{k}^{Z} Z\left(t_{k}\right)$, where $X\left(t_{k}^{+}\right)$is the value of the concentration $X$ after division at instant $t_{k} ; \lambda_{k}^{X}>0, \lambda_{k}^{Y}>0$ and $\lambda_{k}^{Z}>0$ are parameters.

The cell division cycle can be larger than the period of oscillations $T$ [23] or similar, as in proliferating human cells [27] (where circadian clock is a major synchronizing factor, which orchestrates daily rhythms regulating the cell division cycle), or two times faster, as in cyanobacteria [28]. The values $\lambda_{k}^{X}, \lambda_{k}^{Y}, \lambda_{k}^{Z}$ have been selected around 0.5 in [16], but in [29] it has been observed in vivo that concentrations do not jump significantly after cell division. In the present work we will adopt the latter hypothesis by taking $\lambda_{k}^{X}, \lambda_{k}^{Y}$, $\lambda_{k}^{Z}$ close to 1 .

The modeling of such a hybrid oscillator corresponds to a mother cell in the population, then after each division the daughter cells have a similar dynamics and forthcoming divisions augment the population. It is assumed that division instants $t_{k}$ for each cell are different, then study of phase synchronization behavior in a population (assuming that there is no interconnection between cells) can be analyzed using (1). If the phase converges to a steady-state in this hybrid system under some conditions, then the population will be phase synchronized in some sense.

Taking parameter values $c_{1}=c_{3}=c_{5}=0.3, c_{2}=c_{4}=$ $c_{6}=0.15, n=10$ and $K=1$, the period of the autonomous oscillation of (1) is obtained as $T=26.07 \mathrm{~min}$. With these values of parameters and for the case $u(t)=0$ and $t_{k}=k T-v_{k}, k \geq$ 1 , where $v_{k} \in[0.15 T, 0.30 T]$ is a uniformly distributed random variable, the results of the Goodwin oscillator simulation for the same initial conditions and different realizations of $v_{k}$ for 4 different cells undergoing divisions can be seen in Fig. 1. As we can conclude from these results the phase is diverging as it has been noted in [19], [20] and in some experiments of [16]. Next, by taking $u(t)=$ $\max \{0,0.2 \sin (\omega t)\}$ as the common external entraining input and repeating the same experiments, the results are given in Fig. 2. From Fig. 2, it is evident that the oscillations converge to a common entrained mode.

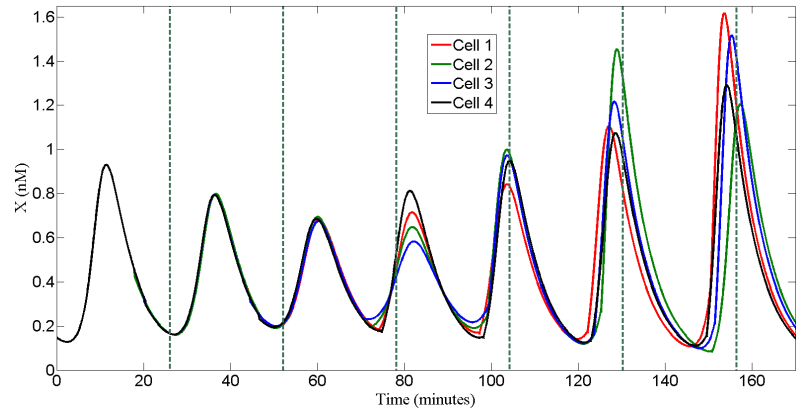

Figure 1. Oscillations of different single cells with cell divisions and without any common input

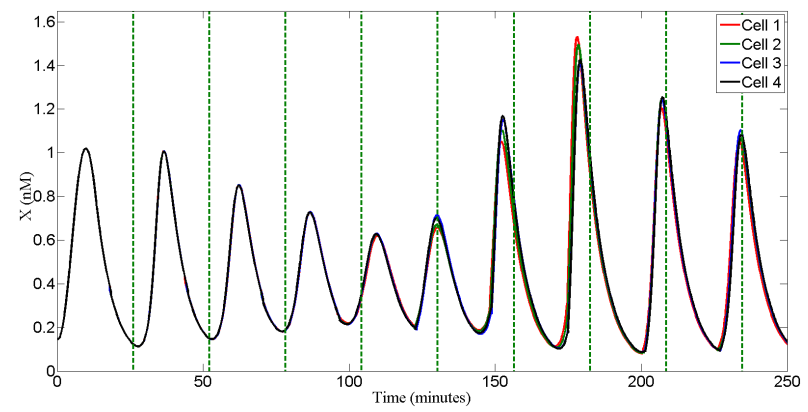

Figure 2. Oscillations of different single cells with cell divisions and common external entraining input

In this paper we will try to find conditions providing both these two types of phase behavior using PRC phase model for small inputs.

\section{Phase MOdel IN VICINITY OF A LIMIT CYCLE}

Details of the standard procedure for a phase model derivation for an oscillator can be found in [3], [4], [13].

\section{A. Linearized model}

Consider a (smooth) dynamical system

$$
\dot{x}=f(x, u), \quad x \in \mathbb{R}^{n}, \quad u \in[-U, U] \subset \mathbb{R}, U>0 .
$$

Denote a solution of (2) with the initial condition $x_{0}$ and input $u$ as $x\left(t, x_{0}, u\right)$ and assume that for $u(t) \equiv 0, t \geq 0$ and some $x_{0} \in \mathbb{R}^{n}$ the system (2) has (non-constant) $T$-periodic solution $x\left(t, x_{0}, 0\right)=$ $\gamma(t)=\gamma(t+T) \in \mathbb{R}^{n}, t \geq 0$. Then the corresponding limit cycle, described by the set $\Gamma=\left\{x \in \mathbb{R}^{n}: x=\gamma(t), 0 \leq t<T\right\}$, attracts a non-empty open bounded set of initial conditions $\mathcal{A} \subset \mathbb{R}^{n}$, $\Gamma \subset \mathcal{A}$, and the linearized system

$$
\begin{aligned}
& \delta \dot{x}(t)=A(t) \delta x(t)+b(t) u(t)+d[\delta x(t), \gamma(t), u(t)], \\
& A(t)=\left.\frac{\partial f(x, u)}{\partial x}\right|_{x=\gamma(t)}, \quad b(t)=\left.\frac{\partial f(x, u)}{\partial u}\right|_{x=\gamma(t)}
\end{aligned}
$$

has $n-1$ multipliers strictly inside the unit cycle and one multiplier equals to 1 [30], [31], where $\delta x(t)=x(t)-\gamma(t)$, the matrix function $A$ and the vector function $b$ are $T$-periodic due to properties of $\gamma$; the function $d[\delta x(t), \gamma(t), u(t)]$ represents the higher order terms with respect to $\delta x(t)$ in the system (2) linearization and for 
all $x \in \mathcal{A}$ and $|u| \leq U$ there exist $d_{1}>0, d_{2}>0$ such that (the function $d$ contains products of $\delta x$ and $u$ with power 2 and higher):

$$
|d(\delta x, \gamma, u)| \leq d_{1}|\delta x|^{2}+d_{2} u^{2} .
$$

Multipliers are the eigenvalues of the monodromy matrix $M=$ $\Phi(T)$ defined via the fundamental matrix function $\Phi$ of the system (3) and the solution of adjoint system $\Psi$ :

$$
\dot{\Phi}(t)=A(t) \Phi(t), \Phi(0)=I ; \dot{\Psi}(t)=-A(t)^{T} \Psi(t), \Psi(0)=I,
$$

where $I$ is the identity matrix and $\Phi(t)^{T} \Psi(t)=I$.

\section{B. Phase variables}

Any point $x_{0} \in \Gamma$ can be characterized by a scalar phase $\varphi_{0} \in[0,2 \pi)$, that uniquely determines the position of the point $x_{0}$ on the limit cycle $\Gamma$ ( $\Gamma$ is a one-dimensional closed curve in $\mathbb{R}^{n}$ ) [3], [4]. The smooth bijective phase map $\vartheta: \Gamma \rightarrow[0,2 \pi)$ assigns to each point $x_{0} \in \Gamma$ the corresponding phase $\varphi_{0}=\vartheta\left(x_{0}\right)$. Any solution of the system (2) $x\left(t, x_{0}, 0\right)$ with $x_{0} \in \Gamma$ satisfies $x\left(t, x_{0}, 0\right)=\gamma\left(t+\varphi_{0} \omega^{-1}\right)$, where $\omega=2 \pi / T$ is the system frequency, provided we choose the convention $\gamma(t)=x\left(t, \vartheta^{-1}(0), 0\right)$, then we can define $\vartheta^{-1}(\varphi)=\gamma\left(\varphi \omega^{-1}\right)$. The phase variable $\varphi: \mathbb{R}_{+} \rightarrow[0,2 \pi)$ is defined for the trajectories $x\left(t, x_{0}, 0\right), x_{0} \in \Gamma$ as $\varphi(t)=\vartheta\left[x\left(t, x_{0}, 0\right)\right]=\vartheta\left[\gamma\left(t+\varphi_{0} \omega^{-1}\right)\right]$. Due to periodic nature of $\gamma(t)$, the function $\varphi(t)$ is also periodic, moreover the function $\vartheta$ can be defined providing $\varphi(t)=\omega t+\varphi_{0}$ and $\dot{\varphi}(t)=\omega$ [3], [4].

The notion of phase can be extended to any solution $x\left(t, x_{0}, 0\right)$ starting in the attraction set $\mathcal{A}$ of the limit cycle. By definition, for all $x_{0} \in \mathcal{A}$ there exists an asymptotic phase $\theta_{0} \in[0,2 \pi)$ such that

$$
\lim _{t \rightarrow+\infty}\left|x\left(t, x_{0}, 0\right)-\gamma\left(t+\theta_{0} \omega^{-1}\right)\right|=0 .
$$

Then there exists the asymptotic phase map $v: \mathcal{A} \rightarrow[0,2 \pi)$ connecting a point $x_{0} \in \mathcal{A}$ and the corresponding phase $\theta_{0}$, i.e. $\theta_{0}=v\left(x_{0}\right)$ and by construction $v\left(x_{0}\right)=\vartheta\left(x_{0}\right)$ for all $x_{0} \in \Gamma$. The asymptotic phase variable $\theta: \mathbb{R}_{+} \rightarrow[0,2 \pi)$ is derived as $\theta(t)=v\left[x\left(t, x_{0}, 0\right)\right], t \geq 0$. In the case $\varphi(t)=\omega t+\varphi_{0}$ we have $\theta(t)=\omega t+\theta_{0}$ and $\dot{\theta}(t)=\omega$, which implies time invariance of this map: if $v\left(x_{1}\right)=v\left(x_{2}\right)$, then $v\left[x\left(t, x_{1}, 0\right)\right]=v\left[x\left(t, x_{2}, 0\right)\right]$ for all $t \geq 0$ and $x_{1}, x_{2} \in \mathcal{A}$ [3]. The initial conditions $x_{1}, x_{2} \in \mathcal{A}$ having the same asymptotic phase determine the isochrone curves [3].

The notion of asymptotic phase variable can be extended to a generic $u(t) \neq 0, t \geq 0$ provided that the corresponding trajectory $x\left(t, x_{0}, u\right)$ stays in the set $\mathcal{A}$ for all $t \geq 0$. In this case the asymptotic phase variable can be defined in a trivial way as $\theta(t)=v\left[x\left(t, x_{0}, u\right)\right], t \geq 0$. Then the variable $\theta\left(t^{\prime}\right)$ at an instant $t^{\prime} \geq 0$ evaluates the asymptotic phase of the point $x\left(t^{\prime}, x_{0}, u\right)$ if one would pose $u(t)=0$ for $t \geq t^{\prime}$. Dynamics of the asymptotic phase variable $\theta(t)$ in the generic case for $u(t) \neq 0, t \geq 0$ is difficult to derive. A local model obtained in a small neighborhood of the limit cycle for infinitesimal inputs is presented below [3], [13].

\section{Infinitesimal PRC}

Consider the case $u(t)=0$ for $t \geq 0$, then by definition $\dot{\gamma}(t)=$ $f(\gamma(t), 0), \ddot{\gamma}(t)=A(t) \dot{\gamma}(t)$ and $\dot{\gamma}(t)=\Phi(t) \dot{\gamma}(0)$ for all $t \geq 0$. Therefore, $\dot{\gamma}(0)=f(\gamma(0), 0)$ is the left eigenvector of the matrix $M$ for the eigenvalue $\lambda_{1}(M)=1$. There exists a right eigenvector $m \in \mathbb{R}^{n}$ such that $m^{T} M=m^{T}$ and $m^{T} \dot{\gamma}(0)=\omega$. Finally, define $Q(t)=m^{T} \Psi(t)^{T}$ then

$$
\begin{aligned}
Q(t) f(\gamma(t), 0) & =m^{T} \Psi(t)^{T} f(\gamma(t), 0) \\
& =m^{T} \Psi(t)^{T} \Phi(t) \dot{\gamma}(0)=m^{T} \dot{\gamma}(0)=\omega
\end{aligned}
$$

From another side, $\theta(t)=v[\gamma(t)]=\omega t+\theta(0)$ and

$$
\omega=\dot{\theta}=\left.\frac{\partial v(x)}{\partial x}\right|_{x=\gamma(t)} f(\gamma(t), 0) .
$$

Therefore $Q(t)=\left.\frac{\partial v(x)}{\partial x}\right|_{x=\gamma(t)}+\zeta(t)$, where $\zeta(t)$ is a row-vector orthogonal to $f(\gamma(t), 0)$. Since $m$ is the eigenvector corresponding to $\lambda_{1}(M)=1$ (or movement on the limit cycle), then $Q(t)=$ $m^{T} \Psi(t)^{T}$ is independent of perturbations orthogonal to the limit cycle flow $f(\gamma(t), 0)$ and the convention

$$
Q(t)=\left.\frac{\partial v(x)}{\partial x}\right|_{x=\gamma(t)}=m^{T} \Psi(t)^{T}
$$

is adopted. The first equality in (4) explains the physical meaning of $Q(t)$, while the last equality in (4) is used for numerical calculation. The function $Q(t)$ is $T$-periodic by construction.

The function $Q\left(\phi \omega^{-1}\right)$ for phase $\varphi \in[0,2 \pi)$ is called infinitesimal PRC [3], it serves as the phase response characteristics for a delta-impulse input.

\section{Phase dynamics}

Consider the case $u(t) \neq 0$ (we assume that $x\left(t, x_{0}, u\right) \in \mathcal{A}$ for all $t \geq 0$ ), then

$$
\begin{aligned}
\dot{\theta}(x(t))= & \dot{\theta}(\gamma(t)+\delta x(t)) \\
= & \left.\frac{\partial v(x)}{\partial x}\right|_{x=\gamma(t)+\delta x(t)} f(\gamma(t)+\delta x(t), u(t)) \\
= & \left.\frac{\partial v(x)}{\partial x}\right|_{x=\gamma(t)} f(\gamma(t), u(t)) \\
& +r_{1}[\gamma(t), \delta x(t), u(t)]^{T} \delta x(t)
\end{aligned}
$$

where the term $r_{1}[\gamma(t), \delta x(t), u(t)]^{T} \delta x(t)$ corresponds to the powers of $\delta x(t)$ higher than one in the Taylor series of the function $\dot{\theta}(\gamma(t)+\delta x(t))$ with respect to the variable $\delta x(t)$. From above, the quantity $\dot{\theta}(t)=\omega$ should be satisfied for $u(t)=0$, therefore $\left.\frac{\partial v(x)}{\partial x}\right|_{x=\gamma(t)} f(\gamma(t), 0)+r_{1}[\gamma(t), \delta x(t), 0]^{T} \delta x(t)=\omega$, which implies the property $r_{1}[\gamma(t), \delta x(t), 0)=0$. Next,

$$
\begin{aligned}
\dot{\theta}(x(t))= & \left.\frac{\partial v(x)}{\partial x}\right|_{x=\gamma(t)} f(\gamma(t), 0) \\
& +\left.\left.\frac{\partial v(x)}{\partial x}\right|_{x=\gamma(t)} \frac{\partial f(\gamma(t), u)}{\partial u}\right|_{u=0} u(t) \\
& +g[\gamma(t), \delta x(t), u(t)], \\
g(\gamma, \delta x, u)= & r_{1}(\gamma, \delta x, u)^{T} \delta x+r_{2}(\gamma, u) u^{2}
\end{aligned}
$$

where $r_{2}(\gamma, u) u^{2}$ represents the terms with powers two and higher for the Taylor series of the function $\left.\frac{\partial v(x)}{\partial x}\right|_{x=\gamma(t)} f(\gamma(t), u(t))$ with respect to the control $u$. For all $x \in \mathcal{A}$ and $|u| \leq U$ there are $g_{1}>0$ and $g_{2}>0$ such that $|g(\gamma, \delta x, u)| \leq g_{1} u^{2}+g_{2}|\delta x|^{2}$. Recalling the previously introduced designations we obtain

$$
\dot{\theta}=\omega+Q(t) b(t) u(t)+g[\gamma(t), \delta x(t), u(t)] .
$$

This model has been derived around the solution $\gamma(t)$, due to the periodicity of the solution $\gamma\left(t+\phi \omega^{-1}\right), \phi \in[0,2 \pi)$ and $u$, the model for $\gamma\left(t+\phi \omega^{-1}\right)$ has a similar form [3], [13]:

$$
\begin{aligned}
\dot{\theta}= & \omega+Q\left(t+\phi \omega^{-1}\right) b\left(t+\phi \omega^{-1}\right) u(t) \\
& +g\left[\gamma\left(t+\phi \omega^{-1}\right), \delta x(t), u(t)\right] .
\end{aligned}
$$


Skipping the residual function $g$ we obtain the first order approximation of the phase model:

$$
\dot{\theta}=\omega+Q\left(t+\phi \omega^{-1}\right) b\left(t+\phi \omega^{-1}\right) u(t) .
$$

Since the property $|g(\gamma, \delta x, u)| \leq g_{1} u^{2}+g_{2}|\delta x|^{2}$ holds for all $x \in \mathcal{A}$ and $|u| \leq U$, such an approximation is rather accurate for a sufficiently small $U$.

Assume that the input $u(t)=w(t)$, where $w(t)$ has a pulse-like form, i.e. $|w(t)| \leq U$ for all $0<t<\mathcal{T}<T$ and $w(t)=0$ for all $t \geq \mathcal{T}$ or $t \leq 0$. Then integration of (5) yields for $t \geq \mathcal{T}$ :

$$
\begin{aligned}
\theta(t)= & \omega t+\theta(0) \\
& +\int_{0}^{t} Q\left(\tau+\theta(0) \omega^{-1}\right) b\left(\tau+\theta(0) \omega^{-1}\right) u(\tau) d \tau \\
= & \omega t+\theta(0)+\operatorname{PRC}[\theta(0)], \\
\operatorname{PRC}(\theta)= & \int_{0}^{\mathcal{T}} Q\left(\tau+\theta \omega^{-1}\right) b\left(\tau+\theta \omega^{-1}\right) u(\tau) d \tau .
\end{aligned}
$$

The map $P R C(\theta), \theta \in[0,2 \pi)$ is defined for the particular pulse $w$ (by definition $-\pi \leq P R C(\theta)<\pi$ for all $\theta \in[0,2 \pi)$ ), it tabulates the phase shift by the pulse $w$ [3], [4]. For $w(t)=\delta(t-\eta)$ with $\eta \in(0, \mathcal{T})$ and $\delta(t)$ is the impulse input, we obtain the infinitesimal PRC

$$
i P R C(\theta)=Q\left(\eta+\theta \omega^{-1}\right) b\left(\eta+\theta \omega^{-1}\right),
$$

which defines the phase shift under an impulse input.

\section{PRC-BASED PHASE MODE FOR AN OSCILLATOR WITH CELL DIVISION}

This section starts with the introduction of the formalized problem statement. Next, the reduced PRC model is introduced and phase synchronization analysis is presented.

\section{A. Problem statement}

Let us consider a population of $N>0$ cells (genetic oscillators) with $s=1,2, \ldots, N$ :

$$
\begin{gathered}
\dot{x}_{s}(t)=f_{s}\left(x_{s}(t), u(t)\right) \quad t \in\left[t_{s, k}, t_{s, k+1}\right), k \geq 0 ; \\
x_{s}\left(t_{s, k+1}^{+}\right)=\Lambda_{s, k} x_{s}\left(t_{s, k+1}\right) \quad k \geq 1,
\end{gathered}
$$

where $x_{s}(t) \in \mathbb{R}^{n}$ is the state (concentrations of different products) of $s^{\text {th }}$ cell and the input $u(t)$ is a periodical train of pulses

$$
u(t)=\sum_{i=0}^{+\infty} w(t-i \mathbb{T}),
$$

with a pulse $w(t), w(t)=0$ for all $t \geq \mathcal{T}$ or $t \leq 0$, $\sup _{0 \leq t \leq \mathcal{T}}|w(t)| \leq W<+\infty$ and $\mathcal{T}<\mathbb{T}$ where $\mathbb{T}>0$ is the period of $u ; t_{s, 0}=0$ and $t_{s, k}, k \geq 0$ is a strictly increasing sequence of impulses (discontinuous jumps in (7)) for all $s=1,2, \ldots, N, \Lambda_{s, k}=\operatorname{diag}\left[\lambda_{s, k, 1}, \ldots, \lambda_{s, k, n}\right] \in \mathbb{R}^{n \times n}$ with $\lambda_{s, k, 1} \in[1-\epsilon, 1]$ for some $\epsilon>0$ sufficiently small. The periodical input $u(t)$ models common entrainment for all cells and discontinuities at instants $t_{s, k}, k \geq 1$ represent the cell division, the diagonal matrix $\Lambda_{s, k}$ determines changes in the state vector (in concentrations) after division. The instants of division $t_{s, k}$ and concentration changes $\Lambda_{s, k}$ may be different for each cell.

Remark 1. Note that formally at each $t_{s, k}, k \geq 1$ the population should be augmented by a daughter cell, that has dynamics similar to mother one. Then the number $N$ is continuously growing. In the present work, we will consider a fixed size of the population $N$, since as it will be shown below (and due to similarity of dynamics for newborn cells and initial conditions) the problem of phase synchronization can be analyzed using the model even a single cell.

It is necessary to establish conditions (restrictions on $f_{s}, u$ and $t_{s, k}$ or $\Lambda_{s, k}$ ) under which there exists a synchronization phenomenon in the cell population (7).

\section{B. Reduced phase model under cell division}

The presence of divisions can be alternatively modeled by an additional impulsion input:

$$
\dot{x}_{s}(t)=f_{s}\left(x_{s}(t), u(t)\right)+\sum_{k=1}^{+\infty} \chi_{s, k} \delta\left(t-t_{s, k}\right),
$$

where $\delta(t)$ is delta-impulse function, $\chi_{s, k}=\left[\Lambda_{s, k}-I_{n_{s}}\right] x_{s}\left(t_{s, k+1}\right)$ and $I_{n_{s}}$ is the identity matrix of dimension $n_{s} \times n_{s}, s=1,2 \ldots N$.

Assumption 1. [24] For each $s=1,2, \ldots, N, s^{\text {th }}$ subsystem in (7), with $u=0$ and $\Lambda_{s, k}=I_{n_{s}}$ for all $k \geq 0$, has a limit cycle $\Gamma_{s} \subset \mathcal{A}_{s}$ with an open set of attraction $\mathcal{A}_{s} \subset \mathbb{R}^{n_{s}}$, and with period $T_{s}>0, \omega_{s}=2 \pi T_{s}^{-1}$.

This assumption says that each cell in the population, if there is no entrainment $u$ and cell division, is an oscillator with the limit cycle $\Gamma_{s}$ and period $T_{s}$. Under Assumption 1 and using the theory presented in Section III, for each cell in (7) it is possible to define its asymptotic phase $\theta_{s} \in[0,2 \pi)$. Under additional restrictions that $\epsilon$ and $W$ are sufficiently small, we can design a phase dynamical model of (7) in some vicinity of $\Gamma_{s}$ as in Section III. Since the model derived in (5) is based on the first order approximation and in the system (8) there are two inputs ( $u$ and the train of impulses), by superposition principle (5) takes the form in this case for $s=$ $1,2, \ldots, N$ :

$$
\begin{aligned}
\dot{\theta}_{s}= & \omega_{s}+Q_{s}\left(t+\theta_{s, 0} \omega_{s}^{-1}\right) b_{s}\left(t+\theta_{s, 0} \omega_{s}^{-1}\right) u(t) \\
& +Q_{s}\left(t+\theta_{s, 0} \omega_{s}^{-1}\right) \sum_{k=1}^{+\infty} \chi_{s, k} \delta\left(t-t_{s, k}\right),
\end{aligned}
$$

where $b_{s}\left(t+\theta_{s, 0} \omega_{s}^{-1}\right)=\left.\frac{\partial f_{s}\left(x_{s}, u\right)}{\partial u}\right|_{x_{s}=\gamma_{s}\left(t+\theta_{s, 0} \omega_{s}^{-1}\right)}$ and $\gamma_{s}(t+$ $\left.\theta_{s, 0} \omega_{s}^{-1}\right)$ is a trajectory of $s^{\text {th }}$ cell in (7) for $u=0$ and $\Lambda_{s, k}=$ $I_{n_{s}}$ for all $k \geq 0$ with initial conditions in $\Gamma_{s}$ with the initial phase $\theta_{s, 0} \in[0,2 \pi], Q_{s}(t)$ is the infinitesimal PRC derived in Section III. This model is constructed around the base trajectory $\gamma_{s}\left(t+\theta_{s, 0} \omega_{s}^{-1}\right)$ under the assumption that the perturbed trajectory with $u \neq 0$ and $\Lambda_{s, k} \neq I_{n_{s}}$ stays close to that one [13]. Since such a closeness assumption is rather restrictive and may be invalid on a sufficiently long time interval (the excited trajectory can belong to a small vicinity of $\Gamma_{s}$ for sufficiently small $\epsilon$ and $W$, but moving away from $\gamma_{s}\left(t+\theta_{s, 0} \omega_{s}^{-1}\right)$ due to phase shift induced by external inputs), then it is better to recalculate the phase of base trajectory $\gamma_{s}(t+$ $\theta_{s, 0} \omega_{s}^{-1}$ ) after a period $\mathbb{T}$, for example (that is the idea of Poincaré phase map approach [3]). In this case by recurrent integration of (9) (similarly as for (6)) the phase shift over interval $[i \mathbb{T},(i+1) \mathbb{T}]$ can be evaluated as follows

$$
\begin{gathered}
\theta_{s, i+1}=\omega_{s}\left(\mathbb{T}-T_{s}\right)+\theta_{s, i}+\operatorname{PR} C_{s}\left(\theta_{s, i}\right)+\Delta_{s, i} \\
P R C_{s}(\theta)=\int_{0}^{\mathcal{T}} Q_{s}\left(t+\theta \omega_{s}^{-1}\right) b_{s}\left(t+\theta \omega_{s}^{-1}\right) w(t) d t \\
\Delta_{s, i}=\sum_{k \in \mathbb{K}_{s, i}} \chi_{s, k} Q_{s}\left(t_{s, k}+\theta_{s, i} \omega_{s}^{-1}\right)
\end{gathered}
$$


for all $s=1,2, \ldots, N$, where $\mathbb{K}_{s, i}=\left\{k \geq 1: t_{s, k} \in[i \mathbb{T},(i+\right.$ 1) $\mathbb{T}]\}$ is the set of indexes whose impulses happen in the interval $[i \mathbb{T},(i+1) \mathbb{T}], P R C_{s}:[0,2 \pi) \rightarrow[0,2 \pi)$ is the PRC of $s^{\text {th }}$ oscillator for the pulse $w, \Delta_{s, i} \in \mathbb{R}$ is the phase perturbation imported by cell division on the interval $[i \mathbb{T},(i+1) \mathbb{T}]$.

Remark 2. Formally the set $\mathbb{K}_{s, i}$ can be decomposed on two parts:

$$
\begin{gathered}
\mathbb{K}_{s, i}=\mathbb{K}_{s, i}^{1} \cup \mathbb{K}_{s, i}^{2}, \\
\mathbb{K}_{s, i}^{1}=\left\{k \geq 1: t_{s, k} \in[i \mathbb{T}, i \mathbb{T}+\mathcal{T}]\right\}, \\
\mathbb{K}_{s, i}^{2}=[i \mathbb{T}+\mathcal{T},(i+1) \mathbb{T}],
\end{gathered}
$$

where $\mathbb{K}_{s, i}^{1}$ characterizes the impulses arrived for $u(t) \neq 0$ and $\mathbb{K}_{s, i}^{2}$ is for $u(t)=0$ on the interval $[i \mathbb{T},(i+1) \mathbb{T}]$. Then the model (10) can be rewritten as follows

$$
\begin{gathered}
\theta_{s, i+1}=\omega_{s}\left(\mathbb{T}-T_{s}\right)+\theta_{s, i}+P R C_{s}\left(\theta_{s, i}+\Delta_{s, i}^{1}\right)+\Delta_{s, i}, \\
\Delta_{s, i}=\Delta_{s, i}^{1}+\Delta_{s, i}^{2}, \\
\Delta_{s, i}^{j}=\sum_{k \in \mathbb{K}_{s, i}^{j}} \chi_{s, k} Q_{s}\left(t_{s, k}+\theta_{s, i} \omega_{s}^{-1}\right), j=1,2 .
\end{gathered}
$$

The difference with respect to (10) is that the perturbation caused by cell division appears nonlinearly in the last model. For brevity of consideration only the case of (10) is studied below.

\section{Phase synchronization}

The model (10) for each $s=1,2, \ldots, N$ is a scalar nonlinear integrator-like discrete-time system (that is a big advantage with respect to (7)) with the state $\theta_{s, i}$ and external input $\omega_{s}\left(\mathbb{T}-T_{s}\right)+$ $\Delta_{s, i}$, where the constant part represents influence of entrainment and $\Delta_{s, i}$ is the perturbation originated by cell division.

Assume that there is no common entrainment and $u(t)=0$, then the model (10) can be simplified to a pure integrator over the interval $[i \mathbb{T},(i+1) \mathbb{T}]$ :

$$
\theta_{s, i+1}=\theta_{s, i}+\Delta_{s, i} .
$$

If $\Delta_{s, i}$ are different for each $s=1,2 \ldots N$ and have not a zero mean, then the phase $\theta_{s, i}$ will be drifting in a unique manner for each $s=1,2 \ldots N$. Thus, there is no phase synchronization, it is the case presented in Fig. 1 of Section II and also observed in [20], [19].

Therefore, the synchronous properties of (10) depend critically on the nonlinear function $P R C_{s}$. In this work, as in [10], [11], [13], we assume that the PRC map has particular properties (similar to type II PRC from [32]).

Assumption 2. [24] For all $s=1,2, \ldots, N$ the map $P R C_{s}$ is continuously differentiable and there exist $0<\beta_{s} \leq 1$ and $\Theta_{s} \in$ $[0,2 \pi)$ such that the equation $P R C_{s}\left(\theta_{s}^{0}\right)=\omega_{s}\left(T_{s}-\mathbb{T}\right)$ has a solution $\theta_{s}^{0} \in[0,2 \pi)$ with

$$
-2+\beta_{s} \leq \frac{\partial P R C_{s}(\theta)}{\partial \theta} \leq-\beta_{s} \quad \forall \theta \in\left[\theta_{s}^{0}-\Theta_{s}, \theta_{s}^{0}+\Theta_{s}\right] .
$$

Obviously, for $\Delta_{s, i}=0$ (no cell division) $\theta_{s}^{0}$ corresponds to a stable equilibrium of the system (10) for given $s$ with the domain of attraction $\left[\theta_{s}^{0}-\Theta_{s}, \theta_{s}^{0}+\Theta_{s}\right][3]$.

Lemma 3. [24] For each $s=1,2, \ldots, N$ under Assumption 2, if $\left|\theta_{s, 0}-\theta_{s}^{0}\right| \leq \Theta_{s}-\beta_{s}^{-1} \overline{\Delta_{s}}$ where $\sup _{i \geq 0}\left|\Delta_{s, i}\right|=\overline{\Delta_{s}}<+\infty$, then

$$
\left|\theta_{s, i}-\theta_{s}^{0}\right| \leq \Theta_{s} \quad \forall i \geq 0, \lim _{i \rightarrow+\infty}\left|\theta_{s, i}-\theta_{s}^{0}\right| \leq \beta_{s}^{-1} \overline{\Delta_{s}} .
$$

Consequently, if cell division influence quantified by $\overline{\Delta_{s}}$ is sufficiently small and initial phase $\theta_{s, 0}$ lies sufficiently close to $\theta_{s}^{0}$, then phase $\theta_{s, i}$ stays in the domain of attraction of $\theta_{s}^{0}$ and asymptotically converge to a vicinity of that equilibrium. Since all cells in population (7) yield this kind of behavior, then under these conditions phases are asymptotically synchronized with the error of synchronization proportional to superposition of $\max \left\{\beta_{s_{1}}^{-1} \overline{\Delta_{s_{1}}}, \beta_{s_{2}}^{-1} \overline{\Delta_{s_{2}}}\right\}$ and $\left|\theta_{s_{1}}^{0}-\theta_{s_{2}}^{0}\right|$ for any $1 \leq s_{1} \neq s_{2} \leq N$.

Theorem 4. [24] Let assumptions 1, 2 be satisfied and $\epsilon, W$ be sufficiently small in (7). If $\left|\theta_{s, 0}-\theta_{s}^{0}\right| \leq \Theta_{s}-\beta_{s}^{-1} \overline{\Delta_{s}}$ for all $s=$ $1,2 \ldots N$, then for any $1 \leq s_{1} \neq s_{2} \leq N$

$$
\begin{gathered}
\left|\theta_{s_{1}, i}-\theta_{s_{2}, i}\right| \leq\left|\theta_{s_{1}}^{0}-\theta_{s_{2}}^{0}\right|+\Theta_{s_{1}}+\Theta_{s_{2}} \quad \forall i \geq 0, \\
\lim _{i \rightarrow+\infty}\left|\theta_{s_{1}, i}-\theta_{s_{2}, i}\right| \leq\left|\theta_{s_{1}}^{0}-\theta_{s_{2}}^{0}\right|+\beta_{s_{1}}^{-1} \overline{\Delta_{s_{1}}}+\beta_{s_{2}}^{-1} \overline{\Delta_{s_{2}}} .
\end{gathered}
$$

This theorem establishes phase-lock behavior in the population (7), which may be composed by different cells. If all cells are identical, then the following synchronization conditions can be obtained.

Corollary 5. [24] Let all conditions of Theorem 4 be satisfied and $P R C_{s}(\theta)=P R C(\theta)$ for all $s=1,2 \ldots, N$ and all $\theta \in[0,2 \pi)$ (then also $\theta_{s}^{0}=\theta^{0}, \Theta_{s}=\Theta$ and $\beta_{s}=\beta$ ). If $\left|\theta_{s, 0}-\theta^{0}\right| \leq$ $\Theta-\beta^{-1} \overline{\Delta_{s}}$ for all $s=1,2 \ldots N$, then

$$
\left|\bar{\theta}_{i}-\theta^{0}\right| \leq \Theta, \quad \forall i \geq 0, \lim _{i \rightarrow+\infty}\left|\bar{\theta}_{i}-\theta^{0}\right| \leq \frac{1}{N \beta} \sum_{s=1}^{N} \overline{\Delta_{s}}
$$

where $\bar{\theta}_{i}=N^{-1} \sum_{s=1}^{N} \theta_{s, i}$ is the average phase of the population.

If there is no cell division, then $\frac{1}{N \beta} \sum_{s=1}^{N} \overline{\Delta_{s}}=0$ ans we recover well-known result on phase synchronization under a periodical entrainment [3], [13].

\section{EXAMPLE}

Next, we illustrate the theoretical results obtained in the previous section.

\section{A. The Goodwin oscillator}

Consider a population of Goodwin oscillators (1). Take all cells in the population identical with the values of parameters given in Section II, then Assumption 1 is satisfied for $T_{s}=T=26.07 \mathrm{~min}$ $\left(\omega=2 \pi T^{-1}\right)$ and select

$$
w(t)= \begin{cases}\max \{0, W \sin (\omega t)\} & \text { if } 0 \leq t \leq T \\ 0 & \text { otherwise }\end{cases}
$$

with $W=0.002$. Setup the same $\Lambda_{s, k, n}$ for all $s$ and $n$ as $\Lambda_{k}$ defined by:

$$
\Lambda_{k}=\operatorname{diag}\left[\begin{array}{lll}
0.99 & 0.98 & 0.98
\end{array}\right]
$$

with $t_{k}=i T-v_{k}, k \geq 1$, where $v_{k} \in[0.15 T, 0.30 T]$ is a uniformly distributed random variable as before. The values $\epsilon$, $W$ are chosen sufficiently small. For this pulse $w(t)$ the obtained $P R C(\theta)$ and $P R C^{\prime}(\theta)=\frac{\partial P R C(\theta)}{\partial \theta}$ are shown in Fig. 3, from these plots $\theta^{0}=2.15, \Theta=1.05$ and $\beta=0.05$, and Assumption 2 is also satisfied. Thus, all conditions of Theorem 4 are verified.

Simulated phase behavior of (1) is shown in Fig. 4 by blue curve (the phase value was computed finding the closest point on the limit cycle at instants $i T$ for $i \geq 0$ and assigning the phase of that point as $\theta_{i}$ ), the values of phase obtained by the model (10) are presented in the same figure by red curve. As we can see, both curves are 


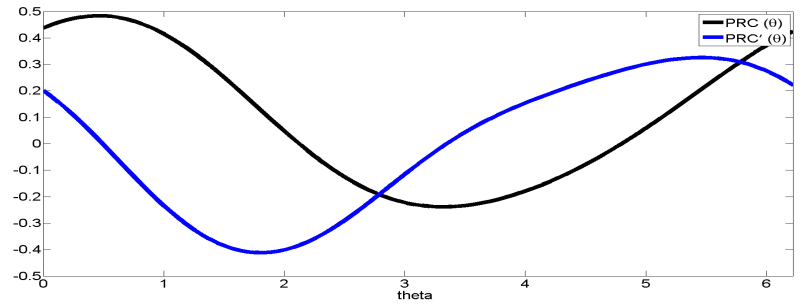

Figure 3. $\operatorname{PRC}(\theta)$ and $\operatorname{PRC}^{\prime}(\theta)$ for the input $w(t)$.

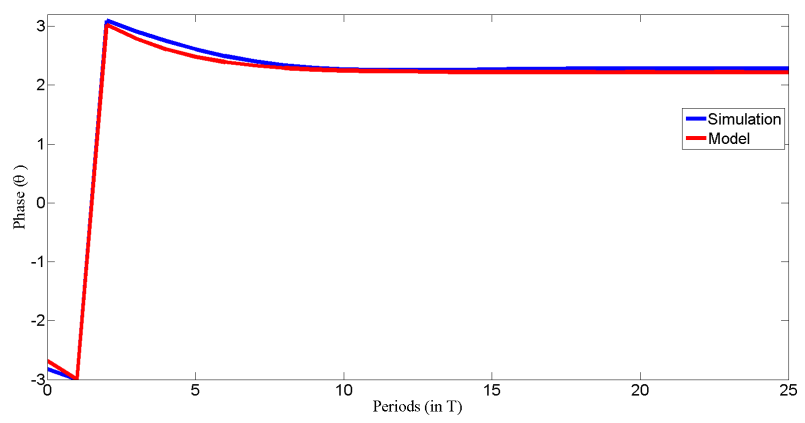

Figure 4. Phase behavior of (1)

very close and that confirms all theoretical developments presented in this work, and phase asymptotically converge to a vicinity of $\theta^{0}$, then synchronization of phase would be observed for a population of Goodwin oscillators (7) as in [16].

\section{CONCLUSION}

The impact of cell division on the dynamics of a population of genetic oscillators is analyzed. Cell division was modeled through impulses, placing the dynamics of population in the class of hybrid systems. As it has been observed in vivo [20], [2], [18], [19] or through simulations [16], oscillations in cells are frequently quite resilient to cell division. Recently this phenomenon has been analyzed by stochastic simulation in [16], where phase synchronization in the population has been observed. In the present work, analytical conditions of phase synchronization are established by applying first order reduced phase model through phase response curve formalism. Theoretical developments proposed in the paper are demonstrated through numerical simulations for two different genetic oscillator models (Goodwin oscillator and Van der Pol oscillator).

\section{REFERENCES}

[1] D. P. King and J. S. Takahashi, "Molecular genetics of circadian rhythms in mammals," Annual review of neuroscience, vol. 23, no. 1, pp. 713-742, 2000.

[2] M. Elowitz and S. Leibler, "A synthetic oscillatory network of transcriptional regulators," Nature, vol. 403, pp. 335-338, 2000.

[3] E. Izhikevich, Dynamical Systems in Neuroscience: The Geometry of Excitability and Bursting. The MIT press, 2007.

[4] A. Pikovsky, M. Rosenblum, and J. Kurths, Synchronization. A Universal Concept in Nonlinear Sciences. Cambridge: Cambridge University Press, 2001.

[5] M. Antle, N. Foley, D. Foley, and R. Silver, "Gates and oscillators II: zeitgebers and the network model of the brain clock," Journal of Biological Rhythms, vol. 22, pp. 14-25, 2007.

[6] G. Zhao, "Phase organization of circadian oscillators in extended gate and oscillator models," J. Theoretical Biology, vol. 264, no. 2, pp. 367-376, 2010.
[7] P. Tass, Phase Resetting in Medicine and Biology. Stochastic Modeling and Data Analysis. Berlin: Springer Verlag, 1999.

[8] C. Canavier and S. Achuthan, "Pulse coupled oscillators and the phase resetting curve," Math Biosci., vol. 226, no. 2, pp. 77-96, 2010.

[9] R. M. Smeal, G. B. Ermentrout, and J. A. White, "Phase-response curves and synchronized neural networks," Philosophical Transactions of the Royal Society B: Biological Sciences, vol. 365, no. 1551, pp. 2407-2422, 2010.

[10] P. Danzl and J. Moehlis, "Spike timing control of oscillatory neuron models using impulsive and quasi-impulsive charge-balanced inputs," in Proc. 29th American Control Conference (ACC2008), Seattle, USA, 2008, pp. 171-176.

[11] D. Efimov, P. Sacre, and R. Sepulchre, "Controlling the phase of an oscillator: a phase response curve approach," in Proc. IEEE CDC 2009, 2009, pp. 7692-7697.

[12] D. Efimov, "Phase resetting control based on direct phase response curve," Journal of Mathematical Biology, vol. 63, no. 5, pp. 855-879, 2011.

[13] — , "Phase resetting for a network of oscillators via phase response curve approach," Biological Cybernetics, vol. 109, no. 1, pp. 95-108, 2015.

[14] L. Glass, Y. Nagai, K. Hall, M. Talajic, and S. Nattel, "Predicting the entrainment of reentrant cardiac waves using phase resetting curves," Physical Rev. E, vol. 65, pp. 65-74, 2002.

[15] W. Govaerts and B. Sautois, "Computation of the phase response curve: A direct numerical approach," Neural Computation, vol. 18, pp. 817847,2006

[16] D. Gonze, "Modeling the effect of cell division on genetic oscillators," Journal of Theoretical Biology, vol. 325, no. 0, pp. 22-33, 2013.

[17] J. Stricker, S. Cookson, M. R. Bennett, W. H. Mather, L. S. Tsimring, and J. Hasty, "A fast, robust and tunable synthetic gene oscillator," Nature, vol. 456, no. 7221, pp. 516-519, 2008.

[18] I. Mihalcescu, W. Hsing, and S. Leibler, "Resilient circadian oscillator revealed in individual cyanobacteria," Nature, vol. 430, pp. 81-85, 2004.

[19] E. Nagoshi, C. Saini, C. Bauer, T. Laroche, F. Naef, and U. Schibler, "Circadian gene expression in individual fibroblasts: Cell-autonomous and self-sustained oscillators pass time to daughter cells," Cell, vol. 119, no. 5, pp. 693-705, 2004.

[20] N. Geva-Zatorsky, N. Rosenfeld, S. Itzkovitz, R. Milo, A. Sigal, E. Dekel, T. Yarnitzky, Y. Liron, P. Polak, G. Lahav, and U. Alon, "Oscillations and variability in the p53 system," Mol. Syst. Biol., vol. 2, p. 0033, 2006

[21] A. Churilov, A. Medvedev, and P. Mattsson, "Periodical solutions in a pulse-modulated model of endocrine regulation with time-delay," IEEE Transactions on Automatic Control, vol. 59, no. 3, pp. 728-733, 2014.

[22] D. Efimov, W. Perruquetti, and A. Shiriaev, "On existence of oscillations in hybrid systems," Nonlinear Analysis: Hybrid Systems, vol. 12, no. 5, pp. 104-116, 2014.

[23] D. S. Tourigny, "Geometric phase shifts in biological oscillators," Journal of Theoretical Biology, vol. 355, no. 0, pp. 239-242, 2014.

[24] H. Ahmed, R. Ushirobira, and D. Efimov, "On robustness of phase resetting to cell division under entrainment," Journal of Theoretical Biology, 2015.

[25] B. Goodwin, "Oscillatory behavior in enzymatic control processes," Adv. Enzyme Regul., vol. 3, pp. 425-438, 1965.

[26] A. Proskurnikov and M. Cao, "Synchronization of goodwins oscillators under boundedness and nonnegativeness constraints for solutions," IEEE Transactions on Automatic Control, vol. PP, no. 99, pp. 1-1, 2016.

[27] S. Bernard and H. Herzel, "Why do cells cycle with a 24 hour period?" Genome Inform., vol. 17, pp. 72-79, 2006.

[28] T. Mori, B. Binder, and C. Johnson, "Circadian gating of cell division in cyanobacteria growing with average doubling times of less than 24 hours," Proc. Natl. Acad. Sci. U.S.A., vol. 93, pp. 10 183-10 188, 1996.

[29] N. Cookson, S. Cookson, L. Tsimring, and J. Hasty, "Cell cycledependent variations in protein concentration," Nucleic Acids Research, vol. 38, no. 8, pp. 2676-2681, 2010.

[30] A. Andronov, A. Vitt, and A. Khaikin, Theory of oscillators, reprint ed Dover, 1987.

[31] V. Yakubovich and V. Starzhinskii, Linear differential equations with periodic coefficients. Wiley, 1975.

[32] D. Hansel, G. Mato, and C. Meunier, "Synchrony in excitatory neural networks," Neural Comput., vol. 7, pp. 307-337, 1995. 